\title{
AN OPTICAL MICROSYSTEM FOR DISPLAYS
}

\author{
Bret Winkler, Dennis Elkins, Allen Tanner \\ Evans \& Sutherland Corporation \\ Salt Lake City, Utah USA \\ Victor Ramsey, Randy Cuff, Leland "Chip" Spangler \\ Aspen Technologies \\ Colorado Springs, Colorado USA
}

\begin{abstract}
This paper describes an optical microsystem for ultra-high pixel count, very large area, laser-illuminated projection displays that address a number of limitations with existing displays. The microsystem consists of CMOS integrated circuits which are flip chip mounted on a surface micromachined MEMS die. This subassembly is mounted into a custom ceramic package and the microstructures are then released using a dry process. Details of the system application, device fabrication, packaging, testing and reliability are presented.
\end{abstract}

\section{INTRODUCTION}

Visual electronic displays are one of the most powerful methods of providing information and entertainment to humans. While most computer displays, televisions and digital theater projectors can create satisfactory images with approximately one million pixels, displays used in applications such as aircraft flight simulators and planetariums must be able to project very high resolution images over very large areas. Some simulation systems require eye-limited resolution. This requires systems that can display 32 million or more, full-color, grey scale pixels at high refresh rates.

Projection displays have been manufactured for a number of years using conventional Cathode Ray Tube (CRT) technology [1]. Up to eight of these CRT-based projectors must be located around the periphery of the display screen to achieve high resolution images over the very large area. These individual projectors must then be spatially, temporally and chromatically synchronized, creating significant image processing requirements along with significant overhead to resynchronize the system on a regular basis. These distributed display systems also create challenges for installation and they are subject to limitations in brightness, contrast ratio and chromatic intensity.

More recently, Liquid Crystal Display (LCD) and MEMSbased projectors using Digital Mirror Device (DMD) technology [2] have been introduced with subsequent improvement in optical performance. High pixel count systems based on these technologies still require several projectors to be placed around the image screen however, and thus are subject to the same synchronization and installation issues that CRT-based systems have. Since CRT, LCD and DMD-based displays are twodimensional arrays, in order to increase the size of the display and maintain spatial resolution, the number of pixels must increase by a squared factor. Projectors that use these technologies thus have practical limitation of a couple million pixels.

To address the limitations inherent in these two-dimensional projectors, a system that uses a linear array of pixels combined with a scanning mirror allows pixel counts to be scaled to much higher levels. A MEMS-based scanning projection display system using a reflective mirror array was proposed by Petersen [3] and subsequently systems based on a linear array of diffractive optical elements [4], [5] have been demonstrated. The Grating Light Valve (GLV) is an example of a MEMS device that uses diffraction to modulate the light intensity in display applications.

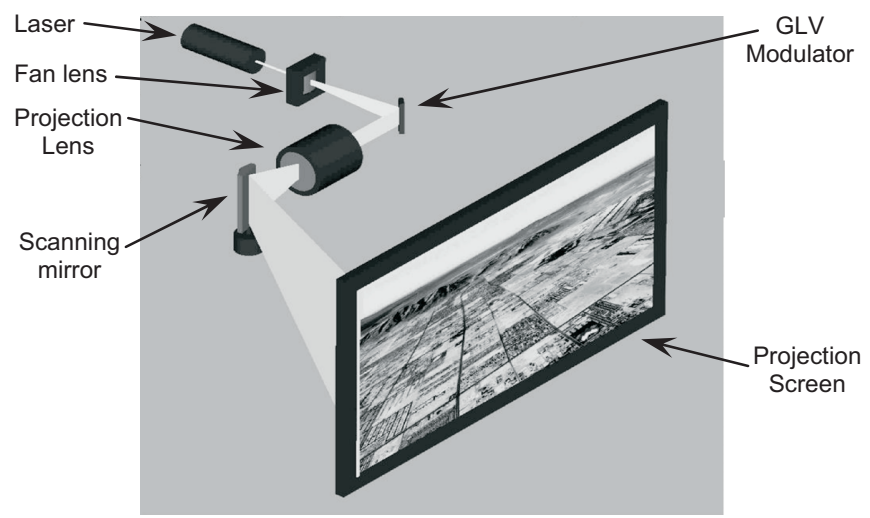

Figure 1. Projection display system based on a GLV modulator, a laser and a scanning mirror. Actual displays combine signals from a red, green and blue laser and three GLV modulators to create the full color display.

To create ultra-high resolution, large area, scanned mirror displays, not only does the number of pixels have to increase but the switching speed of the pixels must be increased. The need for high switching speed in these high resolution, scanned projector systems precludes the use of mirror arrays such as the DMD which are generally too slow.

\section{SYSTEM AND DEVICE DESIGN}

This paper presents a GLV-based optical microsystem that is a key component in a scanned laser projection display that addresses limitations of existing display technologies while providing greater system flexibility and improved optical performance with higher contrast ratio, brightness and finer resolution. At the same time, the system consumes lower power and offers lower system installation and maintenance costs. The display is designed to project a full-color, 16-bit grey scale, 48 bits per pixel, $\sim 32$ million pixel (4,096 rows x 8,192 columns) images at a $60 \mathrm{~Hz}$ refresh rate over very large areas.

The system (Figure 1) employs a custom graphics processor and optical projection system along with custom lasers at three different wavelengths, red $(448 \mathrm{~nm})$, green $(532 \mathrm{~nm})$ and blue $(631 \mathrm{~nm})$. The custom lasers each project about 6 to 12 watts of optical power through a lens system that fans the light into a line and focuses it onto one of three GLV modulators at an angle of about 10 degrees to the optical axis. The diffracted light is then 
selected and focused onto a scanning mirror and additional lens systems project this light onto the display screen. Data is provided to the GLV modulator synchronously with the scanning mirror to sweep the image over the display screen.

The GLV modulator consists of a linear array of clampedclamped silicon nitride beams which are suspended over a conductive surface. The beams have a top reflective surface of aluminum and are 3.2 microns wide on a 3.7 micron pitch. The beams are about 200 microns long between the anchors.

Alternate beams are connected to a common "bias" potential while the other beams are individually connected to signal lines. The active beams are deflected by the potential that is applied to them relative to the conducting layer below the beams. When the potential on the bias and active beams is the same, the pixel is in the reflective state and no light is coupled into the projection optics (Figure 2a). When the active beams are brought to $1 / 4$ of a wavelength below the bias beams (Figure 2b), all of the light from the both the +1 and -1 diffracted order is captured by the projection optics. Light from the other diffracted orders is blocked and not projected.

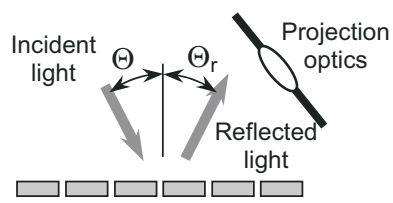

$\sin \left(\Theta_{\mathrm{r}}\right)=\sin \left(\Theta_{\mathrm{i}}\right)$

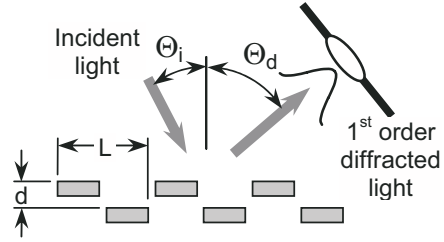

$\sin \left(\Theta_{\mathrm{d}}\right)=\sin \left(\Theta_{\mathrm{i}}\right)-\lambda / \mathrm{L}$ (a) Reflected Dark State

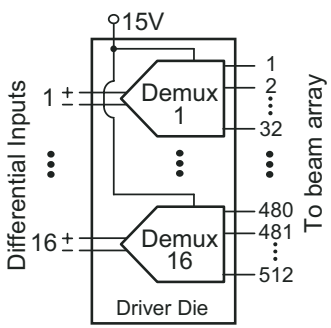

(a) Block diagram of the driver die circuit

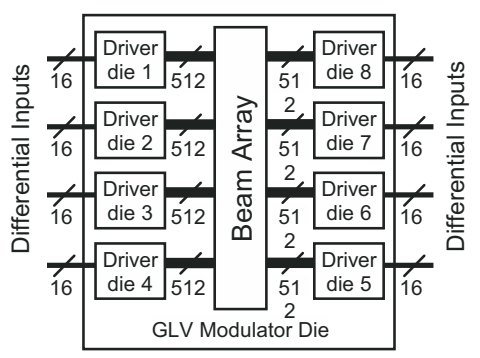

(b) Block diagram of the optical microsystem

Figure 3. Block diagram of the driver die circuit (a) and entire GLV modulator assembly (b).

The 128 analog inputs that are multiplexed into the microsystem are differential signals with a 1 volt swing. A differential input signal path was chosen to help maintain the 16bit analog precision ( $~ 80$ microvolts) that is needed to obtain the desired optical grey scale. This analog data must arrive at a rate of nearly $16 \times 10^{6}$ samples per second per pixel. Once the differential analog signal is demultiplexed, it is level shifted to a 0 to 15 volt single ended line with 200 microvolt resolution. A sample and hold circuit maintains the potential so that it can be clocked onto the active beams with the proper timing. Given the material properties and internal stress in the nitride beams and their physical geometry, 15 volts is sufficient to pull the beams down at least $210 \mathrm{~nm}$ below the bias beams. An analysis of beam mechanics and optical performance is given in [6].

\section{FABRICATION AND PACKAGING}

Figure 2. To project a dark pixel (a), the beams are in the reflective state and the light does not couple into the projection lens. To project a bright pixel (b) the active beams are lowered relative to bias beams so the first order diffractive light couples into the projection optics.

To achieve grey scale, the active beams are held at a position less than $1 / 4$ of the wavelength of the incident light. This acts as a valve that allows some of the light to reflect and some to go into the +1 and -1 orders which means that only a portion of it is coupled into the projection optics. Since 16 bits of grey scale are desired, the active beams in each pixel must be positioned and held with a resolution of $0.002 \mathrm{~nm}$ relative to the bias beams in that pixel.

In order to project 8192 columns at a $60 \mathrm{~Hz}$ refresh rate, the active beam position must be changed in less than 1 microsecond. Since there are 4096 pixels in the device, the 16-bit analog data must be provided to the microsystem at a rate of over $2 \times 10^{9}$ analog data samples (16-bit) per second per color. These specifications were the primary factors in the design of the electrical portion of the device.

Partitioning decisions are important in microsystem design. Size and costs issues make it impractical to use a package that has 4096 input pins to connect to the 4096 pixels. The number of package I/Os can be reduced with time division multiplexing but this requires circuitry to demultiplex the high-speed data prior to applying it to the active beams. A cost/yield analysis has lead to the choice of multiplexing the analog data at a rate of 32:1 which reduces the number of input lines to 128 . The partitioning analysis also lead to the decision to divide the multiplexing task over eight separate pretested "driver" die that are flip chip bonded directly onto the GLV modulator die with the beams. This means that each "driver" die has 16 inputs to control 512 pixels.

The partitioning decisions require two different wafers to be fabricated and assembled in a custom package with a complex assembly process. The driver die wafers are fabricated in a conventional 0.8 micron CMOS process with two metal layers on 6 inch wafers. After probe testing, they are prepared for solder bumping with an electroless $\mathrm{Ni}-\mathrm{Au}$ under bump metallization (UBM) process. These pads are then bumped with eutectic $\mathrm{Pb}-\mathrm{Sn}$ solder to form flip chip interconnections about 80 microns in diameter on a pitch of 200 microns. The bumped wafers are then singulated into die that are $6.9 \mathrm{~mm} \times 5.3 \mathrm{~mm}$ in size.

The GLV modulator die is fabricated on 6 inch wafers with a surface micromachining process that uses a sacrificial layer of polysilicon. The wafer fabrication begins by depositing a thin TiN layer that is patterned to be just under the beam array. A polysilicon sacrificial layer is then deposited over the TiN layer and patterned. Subsequently a thin silicon nitride and a thin layer

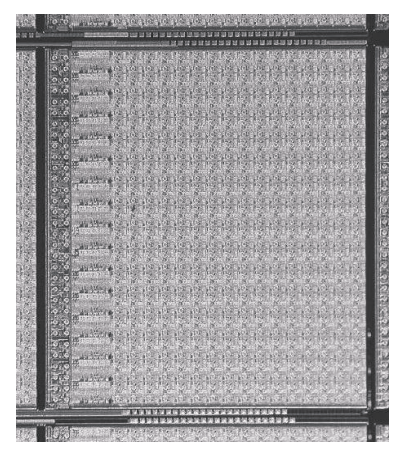

(a)

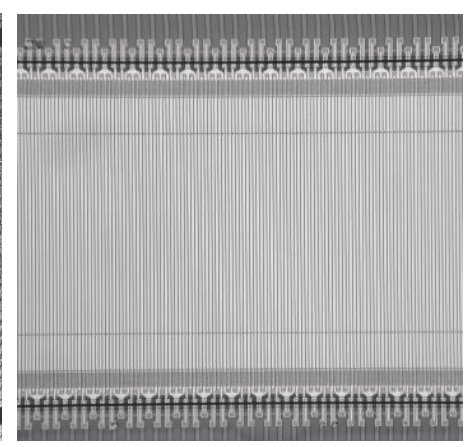

(b)
Figure 4. Photograph of the CMOS driver die (a) and a portion of the beam array on the GLV modulator die (b). 
of aluminum is deposited and patterned to form the beams. Another thicker layer of aluminum is used to connect the aluminum on the beams to the pads that will accept the flipped driver die. This thick aluminum also is used to form the wirebond pads and the interconnection for the driver die inputs.

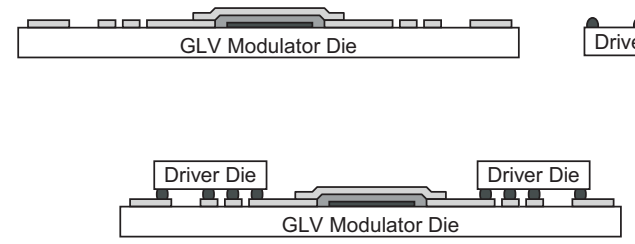

(a)
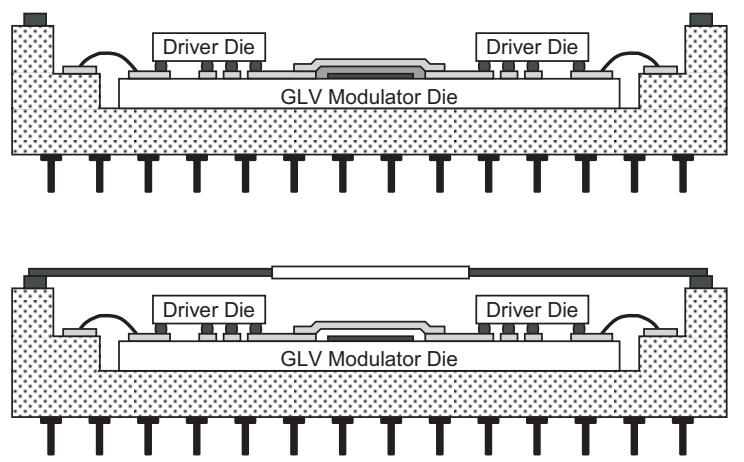

Figure 5. Fabrication and packaging for the optical microsystem. (a) CMOS driver die fabricated and solder bumped and GLV modulator die fabricated with nitride and aluminum beams over a poly-Si sacrificial layer. (b) Driver die are flipped onto the GLV modulator die. (c) The GLV die assembly is packaged (d) The poly-Si sacrificial layer is etched and the windowed lid attached.

After wafer fab, a photoresist layer is patterned and hard baked on the wafer to protect the unreleased beams. This final resist layer serves two functions. Another electroless Ni-Au UBM process is needed, this time on the GLV modulator die, to provide a wettable surface for the driver die flip chip bumps. The final resist layer keeps this metal from being plated on the beams and the wirebond pads. This final resist layer also protects the very thin aluminum on the unreleased beams from being damaged during the saw and clean process. After dicing, this final resist layer is stripped from the GLV die individually using a specially formulated wet processing sequence that preserves the optical properties of the aluminum. The final GLV modulator die size is about $31.8 \mathrm{~mm} \times 19 \mathrm{~mm}$.

To assemble the GLV modulator, the solder bumps on the driver die are coated with a very small amount of solder flux. Eight of these die are aligned and placed on the GLV modulator die. The assembly is then reflowed which allows the solder on the driver die to wet to the adjacent pads on the GLV die forming the desired electrical and mechanical connection. The GLV die assembly is completed by cleaning the flux and underfilling the flipped driver die.

This GLV die assembly is then attached into a custom aluminum nitride (AlN), 470 pin grid array (PGA) package using a "MEMS-grade" adhesive. AlN was chosen for the package because it has much higher thermal conductivity $(\sim 200 \mathrm{~W} / \mathrm{mK})$ and a better CTE match $\left(\sim 4.6 \mathrm{ppm} /{ }^{\circ} \mathrm{C}\right)$ to silicon than conventional alumina ceramic, despite its higher cost and more difficult manufacturing process. The GLV modulator package (Figure 6) has many internal routing layers to allow the input signals to maintain their differential layout and to allow these signals to be fully shielded by internal metal which is tied to ground potential. This conservative design reduces the chance that noise could be coupled onto the sensitive analog input lines. The package has a recessed die cavity with four wirebond tiers. A Kovar seal ring is brazed on the top of the ceramic for subsequent lid attach.

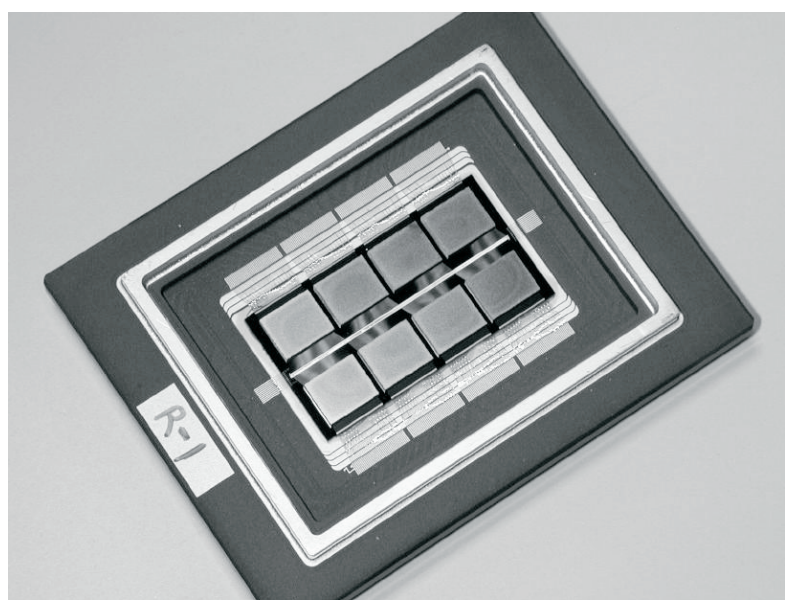

Figure 6. Photograph of the completed optical microsystem without the seam sealed lid. The strip in the middle of the assembly is the linear beam array.

After the GLV modulator assembly is die attached into the AlN package, more than 500 wirebonds are placed on two sides of the die to provide electrical interconnection between the package and the pads on the GLV die assembly. The entire GLV assembly is then placed in a xenon difluoride etch system that allows the $\mathrm{XeF}_{2}$ gas to penetrate the slots between the beams on the GLV modulator die. This releases the beams by selectively etching the underlying polysilicon sacrificial layer (Figure 7). The xenon difluoride does not appreciably attack the other materials used in the assembly. One of the many benefits of this "post-assembly" release process is that there is a reduced chance for microstructure damage during manufacturing.

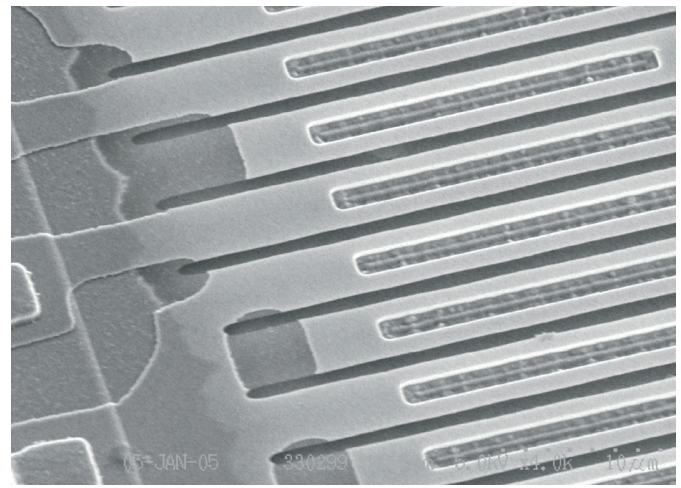

Figure 7. Photograph of the beam ends after etching the sacrificial polysilicon layer in $\mathrm{XeF}_{2}$.

After the beams are released, a Kovar lid with a highly polished glass window is seam sealed onto the seal ring of the PGA package to form a hermetic seal. The glass window has antireflective coatings on both sides. Meeting the detailed optical requirements, including the design of the glass and its installation in the Kovar lid, represented a significant engineering effort to minimize optical distortion. 


\section{TESTING}

After GLV modulator package assembly, the device is mounted to a heat sink which dissipates the power from the driver die and the incident absorbed optical energy. An interface board provides electrical connection and mechanical mounting so it can be precisely aligned. A variety of different test setups is used to evaluate various aspects of device operation. The most basic test involves using a single color laser on an optical bench with the appropriate lenses and optical filters along with a scanning mirror (Figure 8). Full dynamic testing involves more sophisticated image generators and optical setups.

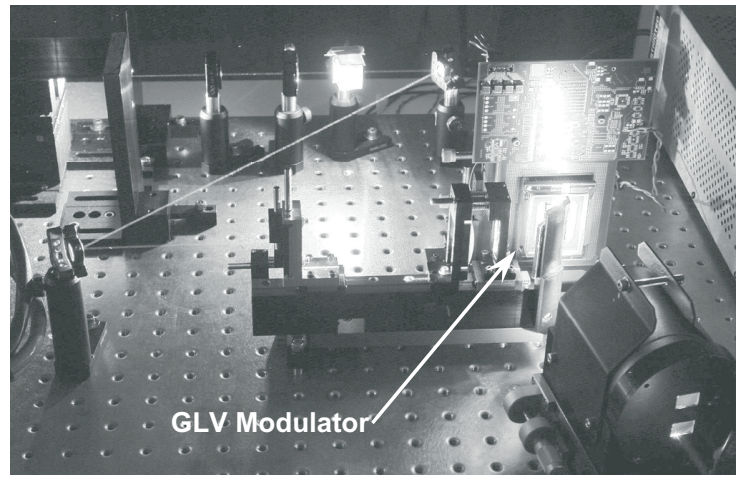

Figure 8. Photograph of the optical microsystem on a test bench being tested with a green laser.

With this simpler setup, the pixels can be cycled from an all "on" to all "off" state by actuating all active beams simultaneously. As shown in Figure 9, the amount of light coupled into the first diffracted order increases as predicted with voltage applied to the active beams. The light intensity peaks at around 13 to 14 volts when the "bias" beams and conductive under layer are held at ground potential. A contrast ratio of greater than 3000:1 is obtained which is a factor of 6 better than other projection displays. The non-linear response is compensated for by the image processing system as it applies the appropriate voltages to achieve a linear grey scale. Since one device design is used in all three color channels, each microsystem must be individually aligned and calibrated in the system.

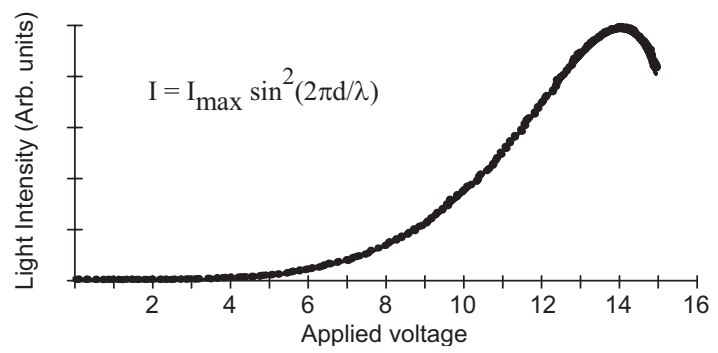

Figure 9. Measured light intensity diffracted into the first order as a function of voltage applied to the active beams [6].

Another advantage of the GLV structure is the very fast pixel response time. This speed is what allows a linear array of elements to be used in conjunction with a scanning mirror. Effectively, each GLV pixel is modulated at a speed that allows it to project an entire row of the display during each refresh cycle. The switching speed for a pixel in this device has been measured to be about $150 \mathrm{nsec}$ from the full on or "bright" state to the full off or "dark" state, but similar devices have been made that switch as fast as $20 \mathrm{~ns}$.
The reliability of displays for these high pixel count applications is critical. While the operating environment is usually confined to indoor conditions, thermal issues due to system-level power dissipation must be considered. Measurement of the resonant frequency of a microstructure is a good way to observe changes in mechanical properties. Figure 10 shows the resonant frequency of an array of beams before and after stress testing that consists of 110 cycles from $30^{\circ} \mathrm{C}$ to $150^{\circ} \mathrm{C}$ and 200 billion actuation cycles on each active beam. The ratio of pre- and post stress resonant frequency is very close to unity suggesting little change over the stress test conditions.

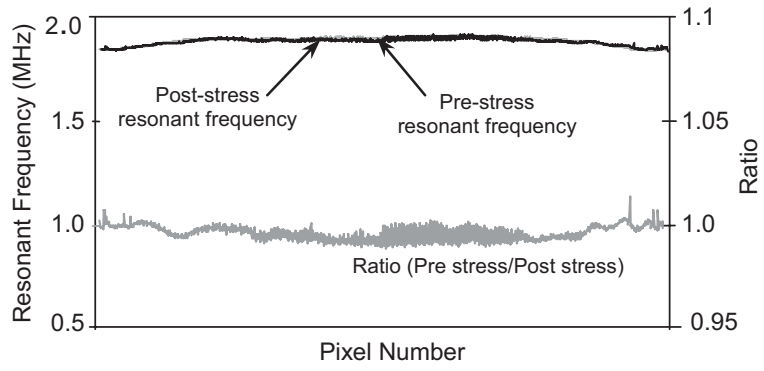

Figure 10. Reliability data on the linear array of beams as measured by the change in resonant frequency after stress testing.

\section{CONCLUSIONS}

An optical microsystem consisting of a linear array of diffractive pixels and analog signal processing circuitry in a custom package has been designed, fabricated, assembled and tested for ultra-high resolution displays. Partitioning analysis including design and technology tradeoffs, yield and time to market considerations has motivated a multiplexed data input strategy along with a multi-die flip chip assembly process. The design takes advantage of the highly selective nature of a dry $\mathrm{XeF}_{2}$ release process to allow the microstructures to be released as one of the last steps in device assembly. The optical microsystem is currently in production and is a key component in a full color, scanning mirror, laser illuminated, 32 million pixel display, the highest resolution MEMS-based display ever made.

\section{REFERENCES}

[1] Evans \& Sutherland Digistar3 laser projector product line (http://www.es.com/products/digital_theater/)

[2] Mignardi, M. A., et. al., "The DMD - An Micro Optical Electromechanical Device for Display Applications," in MEMS and MOEMS Technology and Applications, P. RaiChoudhry, Ed., Bellingham, WA: SPIE Press, 2000, pp.70208.

[3] Petersen, K. E. "Silicon as a Mechanical Material", Proc. IEEE, Vol. 70, No. 5, May 1982, pg 447.

[4] Amm, D.T., Corrigan, R.W., "Grating Light Valve Technology: Update and Novel Applications," Proc. Society of Information Display Symposium, Anaheim, CA, May 19, 1998.

[5] Solgaard, O., Sandejas, F.S.A., and Bloom, D.M. , "Deformable Grating Optical Modulator," Optics Let., No.17, pp 688-691, May, 1992.

[6] Trisnadi, J. I., Carlisle, C.B., and Monteverde, R., "Overview and Applications of Grating Light Valve Based Optical Write Engines for High-Speed Digital Imaging," Proc. Micromachining and Microfabrication Symp., Photonics West 2004, San Jose, CA, Jan. 26, 2004. 